\title{
Event-Triggered Adaptive Backstepping Control for Strict-Feedback Nonlinear Systems with Zero Dynamics
}

\author{
Bo Xu $\mathbb{D}^{1},{ }^{1}$ Xiaoping Liu, ${ }^{1,2}$ Huanqing Wang $\mathbb{D}^{1},{ }^{1}$ and Yucheng Zhou $\mathbb{D}^{1}$ \\ ${ }^{1}$ School of Information and Electrical Engineering, Shandong Jianzhu University, Jinan, China \\ ${ }^{2}$ Faculty of Engineering, Lakehead University, Thunder Bay, ON, Canada P7B 5E1 \\ Correspondence should be addressed to Yucheng Zhou; zhouyucheng@sdjzu.edu.cn
}

Received 23 January 2019; Revised 24 April 2019; Accepted 22 May 2019; Published 10 October 2019

Academic Editor: Xianming Zhang

Copyright (c) 2019 Bo Xu et al. This is an open access article distributed under the Creative Commons Attribution License, which permits unrestricted use, distribution, and reproduction in any medium, provided the original work is properly cited.

\begin{abstract}
This paper focuses on the problem of event-triggered control for a class of uncertain nonlinear strict-feedback systems with zero dynamics via backstepping technique. In the design procedure, the adaptive controller and the triggering event are designed at the same time to remove the assumption of the input-to-state stability with respect to the measurement errors. Besides, we propose an assumption to deal with the problem of zero dynamics. Three different event-triggered control strategies are designed, which guarantees that all the closed-loop signals are globally bounded. The effectiveness of the proposed methods is illustrated and compared using simulation examples.
\end{abstract}

\section{Introduction}

Nowadays, the control problem of uncertain systems draws more and more attention because of the extensive existence in the practical engineering. Due to the fact that vehicles, robots, or UAVs (Unmanned Aerial Vehicles) are required to work more precisely, there have been plenty of researches towards the control problem. For example, Zhou et al. in [1] proposed two adaptive controllers for uncertain nonlinear systems in the presence of input quantization. An eventtriggered output-feedback controller is discussed in [2], for output feedback in switched linear systems. A constrained optimization issue for a class of strict-feedback nonlinear systems is proposed in [3].

Among these researches, adaptive control strategy [4] has been proved to be an effective and efficient method for handling uncertainties and nonlinearities. It introduces a parameter estimator to eliminate the influence caused by unknown parameters. Therefore, over the past few years, there have been many adaptive control strategies for uncertain nonlinear systems. For instance, an adaptive controller is proposed for switched nonlinear systems with coupled input nonlinearities and state constraints in [5]. Sun et al. study a class of state-constrained uncertain nonlinear systems which have steady-state behavior and prescribed transient and design a robust adaptive strategy in [6]. In [7], the authors discuss the problems of adaptive output-feedback tracking control for a class of uncertain nonlinear systems with output constraints, unmodeled dynamics, and quantized input. An extremum seeking controller is proposed based on a fractional-order sliding mode in [8], which has a faster convergence speed. Further, Li et al. propose two adaptive control approaches to synchronize the neural networks in finite time in [9]. A fractional-order sliding mode controller is proposed in [10] for robust stabilization of uncertain fractional-order nonlinear systems. For more applications, see [11-18].

In the practical systems, input energy is obviously limited. Therefore, energy-saving should also be considered in order to extend the systems' lifespan. In the past, the timetriggered scheme is widely used, which means the actuation is executed at every periodic instant even the systems do not need any actuations, and it requires abundant times of experiments to find the most suitable parameters. Therefore, it is impractical for many resource-limited systems. The event-triggered control scheme, which is first proposed in [19], can greatly reduce the control execution times and save the computation costs. In event-triggered systems, actuations 
are not executed periodically, but only when triggering conditions are satisfied. Furthermore, once using the eventtriggered control scheme, the Zeno behavior must be taken into consideration. It is also of great importance to find a way to avoid the Zeno behavior to ensure that the system will not make any mistakes while running. Recently, some event-triggered schemes are proposed to handle different problems. For example, an event-triggered control method is proposed in [20] for parametric strict-feedback nonlinear systems involving backstepping and Lyapunov theory. Xing et al. consider the problems of event-triggered based adaptive control for uncertain nonlinear systems in [21] and propose three event-triggered strategies, while Su et al. design an event-triggered controller by using fuzzy logic method in [22]. In [23], by using input-to-state stable (ISS) condition, a state-feedback event-triggered scheme was proposed and the closed-loop system is guaranteed to be asymptotically stable. Wang et al. design a decentralized event-triggered scheme in [24] for both linear and nonlinear subsystems considering transmission delays. While in networked-control systems, an event-triggered scheme for robust set stabilization is proposed in [25]. What is more, event-triggered schemes also show advantages in consensus and synchronization of multiagent systems in [26-29]. Besides, some results on event-triggered control have also been applied on real systems (see in [30-37]). They extend the range of applications of the event-triggered control scheme.

The zero dynamics, which are well understood and extended to nonlinear finite-dimension systems in [38], are the dynamics of the system when choosing a certain input $u$ that makes output $y$ be identically 0 . It is an expansion of the zero-point concept in linear systems and it is an intrinsic character of nonlinear systems which describes how internal states act when the output is 0 . The stability of zero dynamics in sampled-data nonlinear systems is widely discussed in $[39,40]$, while a funnel controller is proposed in [41] for linear systems considering zero dynamics. For stochastic systems, a controller is designed in [42,43] using event-triggered scheme and backstepping method based on Lyapunov theory. In continuous nonlinear systems, since the zero dynamics are related to the systems dynamics and the minimum interexecution time which is also influenced by the systems dynamics, has to be considered, it increases the complexity to design a controller for such systems. However, to the best of our knowledge, all of the existing event-triggered control strategies were designed without considering zero dynamics in nonstochastic systems. The existence of unstable zero dynamics may restrict the control performance, such as sensitivity, robustness, and stabilization. And the stabilization of zero dynamics in continuous systems is harder to keep than in discrete-time systems [44]. Therefore, it is of great significance to study the stability of zero dynamics for analyzing the internal states of nonstochastic and consensus nonlinear systems.

Inspired by above discussions, an event-triggered based adaptive control is proposed for strict-feedback nonlinear systems with unknown parameters and zero dynamics. Compared with the existing results, the main contributions are given as follows:
(1) The problem of event-triggered adaptive control is first considered for a class of continuous nonlinear systems with zero dynamics. The nonlinearity part in the system does not have to be globally Lipschitz.

(2) Three different triggering conditions are designed, and it is proved that the proposed controllers can make the system asymptotically stable. Then, we prove that every actuation is triggered in finite time, which means the Zeno behavior is successfully avoided.

The rest of the paper is organized as follows. Section 2 proposes the problem formulation and gives the objective of this note. Then, three different design schemes and main theoretical results are described in Section 3. Section 4 gives the simulation results. The conclusion is summarized in Section 5.

\section{Problem Formulation}

Consider a class of strict-feedback nonlinear systems with unknown parameter and zero dynamics:

$$
\begin{aligned}
\dot{\eta} & =f_{0}(\eta)+g_{0}(\eta) x_{1} \\
\dot{x}_{i} & =x_{i+1}+f_{i}\left(\bar{x}_{i}\right)+\varphi_{i}^{T}\left(\bar{x}_{i}\right) \theta, \quad i=1, \ldots, n-1 \\
\dot{x}_{n} & =u(t)+f_{n}\left(\bar{x}_{n}\right)+\varphi_{n}^{T}\left(\bar{x}_{n}\right) \theta \\
y & =x_{1}
\end{aligned}
$$

where $\eta$ denotes the zero dynamics of the system. $f_{0}(\eta)$ and $g_{0}(\eta)$ are known nonlinear functions with respect to $\eta$. And, $x=\left[x_{1}, \ldots, x_{n}\right]^{T} \in \mathbb{R}^{n} \bar{x}_{i}=\left[x_{1}, \ldots, x_{i}\right]^{T} \in \mathbb{R}^{i}$ are the states of the system, while $u(t): \mathbb{R}^{1} \longrightarrow \mathbb{R}^{1}$ is the system input. $\theta \in \mathbb{R}^{r}$ is a vector which contains all the unknown parameters in the system. $f_{i}(\cdot): \mathbb{R}^{i} \longrightarrow \mathbb{R}^{1}$ and $\varphi_{i}(\cdot): \mathbb{R}^{n} \longrightarrow \mathbb{R}^{r},(i=1, \ldots, n)$ are known nonlinear functions with $\gamma$-th order $(\gamma \geqslant n+1-i)$ smooth with respect to $x_{1}, \ldots, x_{i}$. For convenience, we define $f_{i}(\cdot)$ and $\varphi_{i}(\cdot)$ as $f_{i}$ and $\varphi_{i}$, respectively.

Assumption 1 (see [45]). For the zero dynamics $\dot{\eta}=f_{0}(\eta)+$ $g_{0}(\eta) x_{1}$ in (1), there exists a smooth and definite positive function $V_{\eta}$ and a smooth state-feedback control law $x_{1}=$ $\phi_{0}(\eta)$, with $\phi_{0}(0)=0$, such that

$$
\frac{\partial V_{\eta}}{\partial \eta}\left[f_{0}(\eta)+g_{0}(\eta) \phi_{0}(\eta)\right] \leq-c_{\eta} V_{\eta}
$$

Since system (1) contains the zero dynamics and unknown parameters, the existing control approaches are not suitable. Therefore, the control objective of this work is to design an adaptive law $\dot{\hat{\theta}}$ and control signal $u(t)$ for the system (1), which make the output $y$ asymptotically stable, and all the states in the systems are required to be globally bounded.

\section{Event-Triggered Control Scheme}

Similar to [21], three different adaptive controller design strategies are proposed based on different event-triggered conditions, that is, fixed threshold strategy, relative threshold 
strategy, and switching threshold strategy. In this research, the considered control system is affected by the zero dynamics which exists widely in many practical applications. For each strategy, we start with system (1) using the backstepping technique which is carried out with the help of coordinates transformation:

$$
z_{i}=x_{i}-\alpha_{i-1}
$$

where $\alpha_{i-1}(i=1,2,3, \ldots, n)$ denote the virtual control laws and $z_{i}(i=1,2, \ldots, n)$ are the dynamics of the new coordinates. The design process is started as follows.

Step 1. From the coordinate transformation $z_{1}=x_{1}-\alpha_{0}$, the time derivative of $z_{1}$ is

$$
\begin{aligned}
\dot{z}_{1} & =x_{2}+f_{1}+\varphi_{1}^{T} \theta-\dot{\alpha}_{0} \\
& =z_{2}+\alpha_{1}+f_{1}+\varphi_{1}^{T} \theta-\dot{\alpha}_{0},
\end{aligned}
$$

where $z_{2}=x_{2}-\alpha_{1}$.

Then, choose a Lyapunov function as

$$
V_{1}=V_{\eta}+\frac{1}{2} z_{1}^{2}+\frac{1}{2} \widetilde{\theta}^{T} \Gamma^{-1} \widetilde{\theta}
$$

where $\tilde{\theta}=\theta-\hat{\theta}$ is the estimation error, $\hat{\theta}$ denotes the estimation of the uncertain constant vector $\theta$, and $\Gamma$ denotes a positive definite matrix. Therefore, the derivative of $V_{1}$ is

$$
\begin{aligned}
\dot{V}_{1} \leq & \frac{\partial V_{\eta}}{\partial \eta}\left[f_{0}(\eta)+g_{0}(\eta) x_{1}\right] \\
& +z_{1}\left(z_{2}+\alpha_{1}+f_{1}+\varphi_{1}^{T} \theta-\dot{\alpha}_{0}\right)-\widetilde{\theta}^{T} \Gamma^{-1} \dot{\hat{\theta}} \\
\leq & \frac{\partial V_{\eta}}{\partial \eta}\left[f_{0}(\eta)+g_{0}(\eta) \phi_{0}(\eta)\right] \\
& +\frac{\partial V_{\eta}}{\partial \eta} g_{0}\left[x_{1}-\phi_{0}(\eta)\right] \\
& +z_{1}\left(z_{2}+\alpha+f_{1}+\varphi_{1}^{T} \theta-\dot{\alpha}_{0}\right)-\widetilde{\theta}^{T} \Gamma^{-1} \dot{\hat{\theta}} \\
\leq & \frac{\partial V_{\eta}}{\partial \eta}\left[f_{0}(\eta)+g_{0}(\eta) \phi_{0}(\eta)\right] \\
& +z_{1}\left(z_{2}+\frac{\partial V_{\eta}}{\partial \eta} g_{0}+\alpha_{1}+f_{1}+\varphi_{1}^{T} \theta-\dot{\alpha}_{0}\right) \\
& -\widetilde{\theta}^{T} \Gamma^{-1} \dot{\hat{\theta}}
\end{aligned}
$$

where $\alpha_{0}=\phi_{0}(\eta)$. Next, choose a virtual law as

$$
\alpha_{1}=-c_{1} z_{1}-\frac{\partial V_{\eta}}{\partial \eta} g_{0}-f_{1}-\varphi_{1}^{T} \hat{\theta}+\dot{\alpha}_{0}
$$

where $c_{1}$ is a positive constant. Further, substituting (7) into (6) results in

$$
\dot{V}_{1} \leq-c_{\eta} V_{\eta}-c_{1} z_{1}^{2}+z_{1} z_{2}+\widetilde{\theta}^{T}\left(\tau_{1}-\Gamma^{-1} \dot{\hat{\theta}}\right)
$$

where $\tau_{1}=\varphi_{1}^{T} z_{1}$ is a tuning function.
Step 2. The derivative of $z_{2}$ is given as

$$
\begin{aligned}
\dot{z}_{2}= & \dot{x}_{2}-\dot{\alpha}_{1} \\
= & z_{3}+\alpha_{2}+f_{2}+\varphi_{2}^{T} \theta-\frac{\partial \alpha_{1}}{\partial \eta} \dot{\eta} \\
& -\frac{\partial \alpha_{1}}{\partial x_{1}}\left(x_{2}+f_{1}+\varphi_{1}^{T} \theta\right)-\frac{\partial \alpha_{1}}{\partial \hat{\theta}} \dot{\hat{\theta}}
\end{aligned}
$$

where $z_{3}=x_{3}-\alpha_{2}$.

Then, the Lyapunov function is chosen as $V_{2}=V_{1}+$ $(1 / 2) z_{2}^{2}$. The derivative of $V_{2}$ is

$$
\begin{aligned}
\dot{V}_{2} & =\dot{V}_{1}+z_{2} \dot{z}_{2} \leq \dot{V}_{1}+z_{2}\left(x_{3}+f_{2}+\varphi_{2}^{T} \theta-\dot{\alpha}_{1}\right) \\
& \leq-c_{\eta} V_{\eta}-c_{1} z_{1}^{2}+z_{1} z_{2}+\widetilde{\theta}^{T}\left(\tau_{1}-\Gamma^{-1} \dot{\hat{\theta}}\right)+z_{2}\left[z_{3}\right. \\
& +\alpha_{2}+f_{2}+\varphi_{2}^{T} \theta-\frac{\partial \alpha_{1}}{\partial \eta} \dot{\eta}-\frac{\partial \alpha_{1}}{\partial x_{1}}\left(x_{2}+f_{1}+\varphi_{1}^{T} \theta\right) \\
& \left.-\frac{\partial \alpha_{1}}{\partial \widehat{\theta}} \dot{\hat{\theta}}\right] \leq-c_{\eta} V_{\eta}-c_{1} z_{1}^{2}+\widetilde{\theta}^{T}\left(\tau_{1}-\Gamma^{-1} \dot{\hat{\theta}}\right) \\
& +z_{2}\left[z_{3}+z_{1}+\alpha_{2}+f_{2}-\frac{\partial \alpha_{1}}{\partial \eta} \dot{\eta}-\frac{\partial \alpha_{1}}{\partial x_{1}}\left(x_{2}+f_{1}\right)\right. \\
& \left.+\left(\varphi_{2}^{T}-\varphi_{1}^{T} \frac{\partial \alpha_{1}}{\partial x_{1}}\right) \theta-\frac{\partial \alpha_{1}}{\partial \hat{\theta}}\right] \leq-c_{\eta} V_{\eta}-c_{1} z_{1}^{2} \\
& +\widetilde{\theta}^{T}\left[\tau_{1}-\Gamma^{-1} \dot{\hat{\theta}}+z_{2}\left(\varphi_{2}-\varphi_{1} \frac{\partial \alpha_{1}}{\partial x_{1}}\right)\right]+z_{2}\left[z_{3}\right. \\
& +z_{1}+\alpha_{2}+f_{2}-\frac{\partial \alpha_{1}}{\partial \eta} \dot{\eta}-\frac{\partial \alpha_{1}}{\partial x_{1}}\left(x_{2}+f_{1}\right) \\
& \left.+\left(\varphi_{2}^{T}-\varphi_{1}^{T} \frac{\partial \alpha_{1}}{\partial x_{1}}\right) \widehat{\theta}-\frac{\partial \alpha_{1}}{\partial \hat{\theta}}\right]
\end{aligned}
$$

Similar to Step 1, we choose the virtual law as

$$
\begin{aligned}
\alpha_{2}= & -z_{1}-c_{2} z_{2}-f_{2}+\frac{\partial \alpha_{1}}{\partial \eta} \dot{\eta}+\frac{\partial \alpha_{1}}{\partial x_{1}}\left(x_{2}+f_{1}\right) \\
& -\left(\varphi_{2}^{T}-\varphi_{1}^{T} \frac{\partial \alpha_{1}}{\partial x_{1}}\right) \hat{\theta}+\frac{\partial \alpha_{1}}{\partial \widehat{\theta}} \Gamma\left(\tau_{2}-\varsigma \widehat{\theta}\right)
\end{aligned}
$$

where $c_{2}$ is a positive constant and $\varsigma$ is a positive constant. Further, substituting (11) into (10) results in

$$
\begin{aligned}
\dot{V}_{2} \leq & -c_{\eta} V_{\eta}-c_{1} z_{1}^{2}-c_{2} z_{2}^{2}+z_{2} z_{3} \\
& +z_{2} \frac{\partial \alpha_{1}}{\partial \widehat{\theta}}\left(\Gamma \tau_{2}-\dot{\hat{\theta}}-\Gamma \varsigma \widehat{\theta}\right)+\widetilde{\theta}^{T}\left(\tau_{2}-\Gamma^{-1} \dot{\hat{\theta}}\right)
\end{aligned}
$$

where $\tau_{2}=\tau_{1}+\left(\varphi_{2}^{T}-\left(\partial \alpha_{1} / \partial x_{1}\right) \varphi_{1}^{T}\right) z_{2}$ is a tuning function. 
Step $i(i=3, \ldots, n)$. In this step, we repeat the procedure in a recursive way. The derivative of $z_{i}$ is given as

$$
\begin{aligned}
\dot{z}_{i}= & \dot{x}_{i}-\dot{\alpha}_{i-1} \\
= & x_{i+1}+f_{i}+\varphi_{i}^{T} \theta-\frac{\partial \alpha_{i-1}}{\partial \eta} \dot{\eta} \\
& -\sum_{j=1}^{i-1} \frac{\partial \alpha_{i-1}}{\partial x_{j}}\left(x_{j+1}+f_{j}+\varphi_{j}^{T} \theta\right)-\frac{\partial \alpha_{i-1}}{\partial \hat{\theta}} \dot{\hat{\theta}}
\end{aligned}
$$

Then, the Lyapunov function is chosen as $V_{i}=V_{i-1}+$ $(1 / 2) z_{i}^{2}$, and the derivative of $V_{i}$ is

$$
\begin{aligned}
& \dot{V}_{i}=\dot{V}_{i-1}+z_{i} \dot{z}_{i} \leq \dot{V}_{i-1}+z_{i}\left[x_{i+1}+f_{i}+\varphi_{i}^{T} \theta\right. \\
& \left.-\frac{\partial \alpha_{i-1}}{\partial \eta} \dot{\eta}-\sum_{j=1}^{i-1} \frac{\partial \alpha_{i-1}}{\partial x_{j}}\left(x_{j+1}+f_{j}+\varphi_{j}^{T} \theta\right)-\frac{\partial \alpha_{i-1} \dot{\hat{\theta}}}{\partial \hat{\theta}}\right] \\
& \leq-c_{\eta} V_{\eta}-\sum_{j=1}^{i-1} c_{j} z_{j}^{2}+z_{i-1} z_{i}+\widetilde{\theta}^{T}\left(\tau_{i-1}-\Gamma^{-1} \dot{\hat{\theta}}\right) \\
& +\left(\sum_{j=2}^{i-1} z_{j} \frac{\partial \alpha_{j-2}}{\partial \widehat{\theta}}\right)\left(\Gamma \tau_{i-1}-\dot{\hat{\theta}}-\varsigma \Gamma \widehat{\theta}\right)+z_{i}\left[x_{i+1}+f_{i}\right. \\
& +\varphi_{i}^{T} \theta-\frac{\partial \alpha_{i-1}}{\partial \eta} \dot{\eta}-\sum_{j=1}^{i-1} \frac{\partial \alpha_{i-1}}{\partial x_{j}}\left(x_{j+1}+f_{j}+\varphi_{j}^{T} \theta\right) \\
& \left.-\frac{\partial \alpha_{i-1} \dot{\hat{\theta}}}{\partial \hat{\theta}}\right] \leq-c_{\eta} V_{\eta}-\sum_{j=1}^{i-1} c_{j} z_{j}^{2}+\widetilde{\theta}^{T}\left(\tau_{i-1}-\Gamma^{-1} \dot{\hat{\theta}}\right) \\
& +\left(\sum_{j=2}^{i-1} z_{j} \frac{\partial \alpha_{j-2}}{\partial \widehat{\theta}}\right)\left(\Gamma \tau_{i-1}-\dot{\hat{\theta}}-\varsigma \Gamma \widehat{\theta}\right)+z_{i}\left[x_{i+1}\right. \\
& +z_{i-1}+f_{i}-\frac{\partial \alpha_{i-1}}{\partial \eta} \dot{\eta}-\sum_{j=1}^{i-1} \frac{\partial \alpha_{i-1}}{\partial x_{j}}\left(x_{j+1}+f_{j}\right) \\
& \left.+\left(\varphi_{i}^{T}-\sum_{j=1}^{i-1} \frac{\partial \alpha_{i-1}}{\partial x_{j}} \varphi_{j}^{T}\right) \theta-\frac{\partial \alpha_{i-1} \dot{\hat{\theta}}}{\partial \widehat{\theta}}\right] \leq-c_{\eta} V_{\eta} \\
& -\sum_{j=1}^{i-1} c_{j} z_{j}^{2}+\widetilde{\theta}^{T}\left[\tau_{i-1}-\Gamma^{-1} \dot{\hat{\theta}}\right. \\
& \left.+\left(\varphi_{i}-\sum_{j=1}^{i-1} \frac{\partial \alpha_{i-1}}{\partial x_{j}} \varphi_{j}\right) z_{i}\right]+z_{i}\left[z_{i+1}+\alpha_{i}+z_{i-1}\right.
\end{aligned}
$$

$$
\begin{aligned}
& +f_{i}-\frac{\partial \alpha_{i-1}}{\partial \eta} \dot{\eta}-\sum_{j=1}^{i-1} \frac{\partial \alpha_{i-1}}{\partial x_{j}}\left(x_{j+1}+f_{j}\right) \\
& \left.+\left(\varphi_{i}^{T}-\sum_{j=1}^{i-1} \frac{\partial \alpha_{i-1}}{\partial x_{j}} \varphi_{j}^{T}\right) \hat{\theta}-\frac{\partial \alpha_{i-1}}{\partial \hat{\theta}} \dot{\hat{\theta}}\right] \\
& +\left(\sum_{j=2}^{i-1} z_{j} \frac{\partial \alpha_{j-1}}{\partial \hat{\theta}}\right)\left(\Gamma \tau_{i-1}-\dot{\hat{\theta}}-\varsigma \Gamma \hat{\theta}\right)
\end{aligned}
$$

The virtual law is chosen as

$$
\begin{aligned}
\alpha_{i}= & -z_{i-1}-c_{i} z_{i}-f_{i}+\frac{\partial \alpha_{i-1}}{\partial \eta} \dot{\eta}+\sum_{j=1}^{i-1} \frac{\partial \alpha_{i-1}}{\partial x_{j}}\left(x_{j+1}+f_{j}\right) \\
& +\frac{\partial \alpha_{i-1}}{\partial \hat{\theta}} \Gamma\left(\tau_{i}-\varsigma \widehat{\theta}\right)-\left(\varphi_{i}^{T}-\sum_{j=1}^{i-1} \frac{\partial \alpha_{i-1}}{\partial x_{j}} \varphi_{j}^{T}\right) \hat{\theta} \\
& +\left(\sum_{j=2}^{i-1} z_{j} \frac{\partial \alpha_{j-1}}{\partial \widehat{\theta}}\right) \Gamma\left(\varphi_{i}-\sum_{j=1}^{i-1} \frac{\partial \alpha_{i-1}}{\partial x_{j}} \varphi_{j}\right)
\end{aligned}
$$

where $c_{i}$ is a positive constant. Next, substituting (15) into (14) results in

$$
\begin{aligned}
\dot{V}_{i} \leq & -c_{\eta} V_{\eta}-\sum_{j=1}^{i} c_{j} z_{j}^{2}+z_{i} z_{i+1} \\
& +\left(\sum_{j=2}^{i} z_{j} \frac{\partial \alpha_{j-1}}{\partial \hat{\theta}}\right)\left(\Gamma \tau_{i}-\dot{\hat{\theta}}-\varsigma \Gamma \hat{\theta}\right) \\
& +\widetilde{\theta}^{T}\left(\tau_{i}-\Gamma^{-1} \dot{\hat{\theta}}\right)
\end{aligned}
$$

where $\tau_{i}=\tau_{i-1}+\left(\varphi_{i}^{T}-\sum_{j=1}^{i-1}\left(\partial \alpha_{i-1} / \partial x_{j}\right) \varphi_{j}^{T}\right) z_{i}$ is a tuning function.

In the last step $n, \alpha_{n}=u$, which means the actual control input $u$ appears and it is at our disposal. The objective is now to design an appropriate controller and the triggering event.

3.1. Fixed Threshold Strategy. Under this strategy, the adaptive controller is proposed as

$$
\begin{aligned}
w(t) & =\alpha_{n}-\bar{d} \tanh \left(\frac{z_{n} d}{\varepsilon}\right) \\
\dot{\hat{\theta}} & =\Gamma \tau_{n}-\Gamma \varsigma \widehat{\theta}
\end{aligned}
$$

and the triggering event is given as

$$
\begin{aligned}
& u(t)=w\left(t_{k}\right), \quad \forall t \in\left[t_{k}, t_{k+1}\right) \\
& t_{k+1}=\inf \{t \in \mathbb{R}|| e(t) \mid \geq d\}, \quad t_{1}=0
\end{aligned}
$$

where $e(t)=w(t)-u(t)$ denotes the error between the current control signal and last control signal. $\bar{d}, d$, and $\varepsilon$ are all positive constants with $\bar{d} \geq d . t_{k}, k \in \mathbb{Z}^{+}$is the update time of 
the controller, which means when (20) is triggered, the time will jump to $t_{k+1}$, and the control value $u\left(t_{k+1}\right)$ will be applied to the system. In the period between $t_{k}$ and $t_{k+1}$, the control value will keep being $w\left(t_{k}\right)$ and will not be changed.

Now we analyze the stability of the event-triggered controller in system (1), and the design process to avoid the Zeno behavior is also showed. The theorem and proof are proposed as follows.

Theorem 2. Consider a certain of nonlinear closed-loop system (1) and adaptive controller based on the event-triggered scheme (17)-(20) under Assumption 1. The system is globally stable; that is, all the signals are bounded, and the system can be adjusted by selecting proper parameters. In addition, there must be a time $t^{*}>0$ such that $t^{*}$ is greater than or equal to the execution period $\left\{t_{k+1}-t_{k}\right\}, k \in \mathbb{Z}^{+}$, which means the Zeno behavior is successfully avoided.

Proof. The Lyapunov function is chosen as

$$
V=V_{\eta}+\frac{1}{2} \sum_{i=1}^{n} z_{i}^{2}+\frac{1}{2} \widetilde{\theta}^{T} \Gamma^{-1} \widetilde{\theta}
$$

where $\widetilde{\theta}=\theta-\widehat{\theta}$. During the execution interval $\left[t_{k}, t_{k+1}\right)$, we have $|w(t)-u(t)| \leq d$. Assuming that there exists a continuous time-varying parameter $\sigma(t)$, which satisfies $\sigma\left(t_{k}\right)=0$, $\sigma\left(t_{k+1}\right)= \pm 1$, and $|\sigma(t)| \leq 1, \forall t \in\left[t_{k}, t_{k+1}\right)$, such that $w(t)=u(t)+\sigma(t) d$. For convenience, $\sigma(t)$ and $w(t)$ are defined as $\sigma$ and $w$. With this in mind, we have

$$
\begin{aligned}
\dot{V}= & z_{n} \dot{z}_{n}+\dot{V}_{n-1} \\
\leq & z_{n}\left[u(t)+f_{n}+\varphi_{n}^{T} \theta-\dot{\alpha}_{n-1}\right] \\
& +\left(\sum_{i=2}^{n-1} z_{i} \frac{\partial \alpha_{i-1}}{\partial \widehat{\theta}}\right)\left(\Gamma \tau_{n-1}-\dot{\hat{\theta}}-\Gamma \varsigma \hat{\theta}\right)-\sum_{i=1}^{n-1} c_{i} z_{i}^{2} \\
& +z_{n} z_{n-1}+\widetilde{\theta}^{T}\left(\tau_{n-1}-\Gamma^{-1} \dot{\hat{\theta}}\right)-c_{\eta} V_{\eta}
\end{aligned}
$$

By recalling $w(t)=u(t)+\sigma(t) d$, substituting (17) into (22) gives

$$
\begin{aligned}
\dot{V} & \leq z_{n}\left[\alpha_{n}-\bar{d} \tanh \left(\frac{z_{n} d}{\varepsilon}\right)-\sigma d+f_{n}+\varphi_{n}^{T} \theta\right. \\
& \left.-\dot{\alpha}_{n-1}\right]+\widetilde{\theta}^{T}\left(\tau_{n-1}-\Gamma^{-1} \dot{\hat{\theta}}\right)+\left(\sum_{i=2}^{n-1} z_{i} \frac{\partial \alpha_{i-1}}{\partial \widehat{\theta}}\right) \\
& \cdot\left(\Gamma \tau_{n-1}-\dot{\hat{\theta}}-\Gamma \varsigma \widehat{\theta}\right)-c_{\eta} V_{\eta}-\sum_{i=1}^{n-1} c_{i} z_{i}^{2}+z_{n} z_{n-1} \\
& \leq z_{n}\left[\widetilde{\theta}^{T}\left(\varphi_{n}^{T}-\sum_{i=1}^{n-1} \frac{\partial \alpha_{n-1}}{\partial x_{i}} \varphi_{i}^{T}\right)\right.
\end{aligned}
$$

$$
\begin{aligned}
& \left.+\left(\sum_{i=2}^{n-1} z_{i} \frac{\partial \alpha_{i-1}}{\partial \widehat{\theta}}\right) \Gamma\left(\varphi_{n}^{T}-\sum_{i=1}^{n-1} \frac{\partial \alpha_{n-1}}{\partial x_{i}} \varphi_{i}^{T}\right)\right] \\
& +z_{n}\left[-\bar{d} \tanh \left(\frac{z_{n} d}{\varepsilon}\right)-\sigma d\right]-c_{\eta} V_{\eta} \\
& +\left(\sum_{i=2}^{n-1} z_{i} \frac{\partial \alpha_{i-1}}{\partial \widehat{\theta}}\right)\left(\Gamma \tau_{n-1}-\dot{\hat{\theta}}-\Gamma \varsigma \widehat{\theta}\right)-\sum_{i=1}^{n} c_{i} z_{i}^{2} \\
& +\widetilde{\theta}^{T}\left(\tau_{n-1}-\Gamma^{-1} \dot{\hat{\theta}}\right)+z_{n} \frac{\partial \alpha_{n-1}}{\partial \widehat{\theta}}\left(\Gamma \tau_{n}-\dot{\hat{\theta}}-\Gamma \varsigma \widehat{\theta}\right)
\end{aligned}
$$

Notice that $\tau_{n}-\tau_{n-1}=\left(\varphi_{n}^{T}-\sum_{j=1}^{n-1}\left(\partial \alpha_{n-1} / \partial x_{j}\right) \varphi_{j}^{T}\right) z_{n}$. Then, $\dot{V}$ satisfies

$$
\begin{aligned}
\dot{V} \leq & z_{n}\left[-\bar{d} \tanh \left(\frac{z_{n} d}{\varepsilon}\right)-\sigma d\right]+\widetilde{\theta}^{T}\left(\tau_{n}-\tau_{n-1}\right) \\
& +\left(\sum_{i=2}^{n-1} z_{i} \frac{\partial \alpha_{i-1}}{\partial \widehat{\theta}}\right) \Gamma\left(\tau_{n}-\tau_{n-1}\right) \\
& +\widetilde{\theta}^{T}\left(\tau_{n-1}-\Gamma^{-1} \dot{\hat{\theta}}\right) \\
& +\left(\sum_{i=2}^{n-1} z_{i} \frac{\partial \alpha_{i-1}}{\partial \widehat{\theta}}\right)\left(\Gamma \tau_{n-1}-\dot{\hat{\theta}}-\Gamma \varsigma \widehat{\theta}\right)-\sum_{i=1}^{n} c_{i} z_{i}^{2} \\
& -c_{\eta} V_{\eta}+z_{n} \frac{\partial \alpha_{n-1}}{\partial \widehat{\theta}}\left(\Gamma \tau_{n}-\dot{\hat{\theta}}-\Gamma \varsigma \widehat{\theta}\right) \\
\leq & z_{n}\left[-\bar{d} \tanh \left(\frac{z_{n} d}{\varepsilon}\right)-\sigma d\right]+\widetilde{\theta}^{T}\left(\tau_{n}-\Gamma^{-1} \dot{\hat{\theta}}\right) \\
& +\left(\sum_{i=2}^{n} z_{i} \frac{\partial \alpha_{i-1}}{\partial \widehat{\theta}}\right)\left(\Gamma \tau_{n}-\dot{\hat{\theta}}-\Gamma \varsigma \widehat{\theta}\right)-\sum_{i=1}^{n} c_{i} z_{i}^{2} \\
& -c_{\eta} V_{\eta}
\end{aligned}
$$

Based on the property [46], the hyperbolic tangent function $\tanh (\cdot)$ has

$$
0 \leq|\mu|-\mu \tanh \left(\frac{\mu}{\varepsilon}\right) \leq 0.2785 \varepsilon
$$

where $\varepsilon>0$ and $\mu \in \mathbb{R}$. As above, we design $\dot{\hat{\theta}}$ as $\dot{\hat{\theta}}=\Gamma \tau_{n}-\Gamma \varsigma \hat{\theta}$. Based on the Assumption 1, its derivative is given by

$$
\dot{V} \leq-\sum_{i=1}^{n} c_{i} z_{i}^{2}-c_{\eta} V_{\eta}+\varsigma \widetilde{\theta}^{T} \hat{\theta}+0.2785 \varepsilon
$$

where $\varsigma$ is selected based on [47]. Let $\xi=\min \left\{2 c_{1}\right.$, $\left.\ldots, 2 c_{n}, c_{\eta}, \varsigma / \lambda_{\max }\left(\Gamma^{-1}\right)\right\}$, where $\lambda_{\max }\left(\Gamma^{-1}\right)$ is the maximum eigenvalue of $\Gamma^{-1}$. And with the help of Young's inequalities in [37], $\widetilde{\theta}^{T} \hat{\theta} \leq-(1 / 2) \tilde{\theta}^{T} \widetilde{\theta}+(1 / 2) \theta^{T} \theta$. Finally, let $\Delta_{1}=0.2785 \varepsilon+$ $(\varsigma / 2) \theta^{T} \theta$. Then, we get

$$
\dot{V} \leq-\xi V+\Delta_{1}
$$


Based on the LaSalle's invariance principle in [48] and the result in [46], $z_{1}, \ldots, z_{n}$, and $\hat{\theta}$ are proved to be bounded. Therefore, it can be concluded that the control signal $u(t)$ is also bounded. That is, all the closed-loop signals are globally bounded. Then, assuming that there exists a $t^{*}>0$ such that $\forall k \in \mathbb{Z}^{+},\left\{t_{k+1}-t_{k}\right\} \geq t^{*}$, and noticing $e(t)=w(t)-u(t)$, the following can be verified

$$
\frac{d}{d t}|e|=\frac{d}{d t}(e * e)^{1 / 2}=\operatorname{sign}(e) \dot{e} \leq|\dot{w}|
$$

From (17), we get that $\dot{w}=\dot{\alpha}_{n}-\bar{d} \dot{z}_{n} / \cosh ^{2}\left(z_{n} \bar{d} / \varepsilon\right)$. Since $f_{i}(\cdot)$ and $\varphi_{i}(\cdot)$ are at least $(n+1-i)$ th order smooth functions, $\dot{w}$ is continuous. Next, since $\dot{w}$ is a function of $x$ and $\theta$, and all the closed-loop signals are globally bounded, there must be a constant $\zeta>0$ such that $|\dot{w}| \leq \zeta$. Noticing $e\left(t_{k}\right)=0$ and $\lim _{t \rightarrow t_{k+1}} e(t)=d$, there exists a lower bound of $t^{*}$ which satisfies $t^{*} \geq d / \zeta$; that is, the Zeno behavior is successfully avoided.

3.2. Relative Threshold Strategy. In practical systems, when considering a stabilization problem, the value of the control signal always requires to be considered. In the fixed threshold strategy, no matter how big the control magnitude is, the error $e(t)$ is always bounded by a given constant, which may not be applicable to all practical systems. Based on this consideration, a varying threshold comes to mind. Therefore, the following relative threshold control strategy is proposed:

$$
\begin{aligned}
& w(t) \\
& =-(1+\delta)\left[\alpha_{n} \tanh \left(\frac{z_{n} \alpha_{n}}{\varepsilon}\right)+\overline{d_{1}} \tanh \left(\frac{z_{n} \overline{d_{1}}}{\varepsilon}\right)\right] \\
& \dot{\hat{\theta}}=\Gamma \tau_{n}-\Gamma \varsigma \hat{\theta}
\end{aligned}
$$

and the triggering event is given as

$$
\begin{aligned}
& u(t)=w\left(t_{k}\right), \quad \forall t \in\left[t_{k}, t_{k+1}\right) \\
& t_{k+1}=\inf \left\{t \in \mathbb{R}|| e(t)|\geq \rho| u(t) \mid+d_{1}\right\}, \quad t_{1}=0
\end{aligned}
$$

where $t_{k}, k \in \mathbb{Z}^{+}, \varepsilon, \rho(0<\rho<1), \delta(0<\delta<1), d_{1}$ and $\bar{d}_{1}>$ $d_{1} /(1-\rho)$ are all positive parameters. $d_{1}$ is added specially to guarantee the Zeno behavior will not happen, which will be elaborated in the following proof. Using the control strategy, it reaches the following theorem.

Theorem 3. Consider a certain of nonlinear closed-loop system (1) and adaptive controller based on the event-triggered scheme (29)-(32) under Assumption 1. The system is globally stable; that is, all the signals are bounded, and the system can be adjusted by selecting proper parameters. In addition, there must be a time $t^{*}>0$ such that $t^{*}$ is greater than or equal to the execution period $\left\{t_{k+1}-t_{k}\right\}, k \in \mathbb{Z}^{+}$, which means the Zeno behavior is successfully avoided.

Proof. From (32), we can get that $w(t)=\left(1+\sigma_{1}(t) \rho\right) u(t)+$ $\sigma_{2}(t) d_{1}$ during the interval $\left[t_{k}, t_{k+1}\right)$. Similar to the fixed threshold strategy, $\sigma_{1}(t)$ and $\sigma_{2}(t)$ are time-varying parameters which satisfy $\left|\sigma_{1}(t)\right| \leq 1$ and $\left|\sigma_{2}(t)\right| \leq 1$. Therefore, it reaches that $u(t)=w(t) /\left(1+\sigma_{1}(t) \rho\right)-\sigma_{2}(t) d_{1} /\left(1+\sigma_{1}(t) \rho\right)$. For convenience, $\sigma_{1}(t), \sigma_{2}(t)$, and $w(t)$ are defined as $\sigma_{1}, \sigma_{2}$, and $w$. Then, we get

$$
\begin{aligned}
\dot{V}= & z_{n} \dot{z}_{n}+\dot{V}_{n-1} \\
\leq & z_{n}\left[\frac{w-\sigma_{2} d_{1}}{1+\sigma_{1} \rho}+f_{n}+\varphi_{n}^{T} \theta-\dot{\alpha}_{n-1}\right] \\
& +\left(\sum_{i=2}^{n-1} z_{i} \frac{\partial \alpha_{i-1}}{\partial \widehat{\theta}}\right)\left(\Gamma \tau_{n-1}-\dot{\hat{\theta}}-\Gamma \varsigma \widehat{\theta}\right)-\sum_{i=1}^{n-1} c_{i} z_{i}^{2} \\
& +z_{n} z_{n-1}+\widetilde{\theta}^{T}\left(\tau_{n-1}-\Gamma^{-1} \dot{\hat{\theta}}\right)-c_{\eta} V_{\eta}
\end{aligned}
$$

Since $\forall m \in \mathbb{R}$ and $\varepsilon>0,-m \tanh (m / \varepsilon) \leq 0$, it can be obtained that $z_{n} w \leq 0$. Notice that $z_{n} w /\left(1+\sigma_{1} \rho\right) \leq z_{n} w /(1+$ $\rho)$ and $\left|\sigma_{2} d_{1} /\left(1+\sigma_{1} \rho\right)\right| \leq d_{1} /(1-\rho)$. Therefore, similar to (24), substituting (29) into (33) gives

$$
\begin{aligned}
& \dot{V} \leq \frac{z_{n} w}{1+\rho}+\left|\frac{z_{n} d_{1}}{1-\rho}\right|+z_{n}\left(f_{n}+\varphi_{n}^{T} \theta-\dot{\alpha}_{n-1}\right) \\
& +\left(\sum_{i=2}^{n-1} z_{i} \frac{\partial \alpha_{i-1}}{\partial \widehat{\theta}}\right)\left(\Gamma \tau_{n-1}-\dot{\hat{\theta}}-\Gamma \varsigma \widehat{\theta}\right)-\sum_{i=1}^{n-1} c_{i} z_{i}^{2} \\
& +z_{n} z_{n-1}+\widetilde{\theta}^{T}\left(\tau_{n-1}-\Gamma^{-1} \dot{\hat{\theta}}\right)-c_{\eta} V_{\eta} \\
& \leq z_{n} \alpha_{n}-z_{n} \alpha_{n} \tanh \left(\frac{z_{n} \alpha_{n}}{\varepsilon}\right)+\left|z_{n} \bar{d}_{1}\right| \\
& -z_{n} \bar{d}_{1} \tanh \left(\frac{z_{n} \bar{d}_{1}}{\varepsilon}\right)+\left|\frac{z_{n} d_{1}}{1-\rho}\right| \\
& +z_{n}\left(f_{n}+\varphi_{n}^{T} \theta-\dot{\alpha}_{n-1}\right) \\
& +\left(\sum_{i=2}^{n-1} z_{i} \frac{\partial \alpha_{i-1}}{\partial \widehat{\theta}}\right)\left(\Gamma \tau_{n-1}-\dot{\hat{\theta}}-\Gamma \varsigma \widehat{\theta}\right)-\sum_{i=1}^{n-1} c_{i} z_{i}^{2} \\
& +z_{n} z_{n-1}+\widetilde{\theta}^{T}\left(\tau_{n-1}-\Gamma^{-1} \dot{\hat{\theta}}\right)-z_{n} \alpha_{n}-\left|z_{n} \bar{d}_{1}\right| \\
& -c_{\eta} V_{\eta} \\
& \leq z_{n}\left(\alpha_{n}+f_{n}+\varphi_{n}^{T} \theta-\dot{\alpha}_{n-1}\right)-\left|z_{n} \bar{d}_{1}\right|+\left|\frac{z_{n} d_{1}}{1-\rho}\right| \\
& +\left(\sum_{i=2}^{n-1} z_{i} \frac{\partial \alpha_{i-1}}{\partial \widehat{\theta}}\right)\left(\Gamma \tau_{n-1}-\dot{\hat{\theta}}-\Gamma \varsigma \widehat{\theta}\right)-\sum_{i=1}^{n-1} c_{i} z_{i}^{2} \\
& +z_{n} z_{n-1}+\widetilde{\theta}^{T}\left(\tau_{n-1}-\Gamma^{-1} \dot{\hat{\theta}}\right)+0.557 \varepsilon-c_{\eta} V_{\eta}
\end{aligned}
$$


Then, substituting (15) with $i=n$ into (34) gives

$$
\begin{aligned}
\dot{V} \leq & -\sum_{i=1}^{n} c_{i} z_{i}^{2}-\left|z_{n} \bar{d}_{1}\right|+\left|\frac{z_{n} d_{1}}{1-\rho}\right|+0.557 \varepsilon \\
& +\widetilde{\theta}^{T}\left(\tau_{n}-\Gamma^{-1} \dot{\hat{\theta}}\right) \\
& +\left(\sum_{i=2}^{n} z_{i} \frac{\partial \alpha_{i-1}}{\partial \widehat{\theta}}\right)\left(\Gamma \tau_{n}-\dot{\hat{\theta}}-\Gamma \varsigma \widehat{\theta}\right)-c_{\eta} V_{\eta} \\
\leq & -\sum_{i=1}^{n} c_{i} z_{i}^{2}-c_{\eta} V_{\eta}+\varsigma \widetilde{\theta}^{T} \hat{\theta}+0.557 \varepsilon
\end{aligned}
$$

Similar to (27), it reaches

$$
\dot{V} \leq-\xi V+\Delta_{2}
$$

where $\Delta_{2}=0.557 \varepsilon+(\varsigma / 2) \theta^{T} \theta$. As the proof in Theorem 2, all the closed-loop signals are globally bounded, and the interexecution time $t^{*} \geq\left(\rho|u(t)|+d_{1}\right) / 1 k$, which means the Zeno behavior is successfully avoided.

3.3. Switching Threshold Strategy. In the above, the relative threshold strategy shows its advantages. For example, when the value of control signal $u$ is large, the triggering threshold is large too, which ensures that the system can get longer update intervals, while when the $u$ is close to zero, the system can get precise control. Nevertheless, once the value of control signal $u$ is excessively big, the system will get excessively large errors of control signal. Therefore, the control signal will jump suddenly when an event is triggered, which will give the system a large impulse and affect the performance of the system. Hence, with these considerations, we give the switching threshold strategy as follows:

$$
\begin{aligned}
& u(t)=w\left(t_{k}\right), \quad \forall t \in\left[t_{k}, t_{k+1}\right) \\
& t_{k+1} \\
& = \begin{cases}\inf \left\{t \in \mathbb{R}|| e(t)|\geq \rho| u(t) \mid+d_{1}\right\}, & |u(t)|<\mathrm{D} \\
\inf \{t \in \mathbb{R}|| e(t) \mid \geq d\}, & |u(t)| \geq D,\end{cases}
\end{aligned}
$$

where $D$ is a positive constant parameter designed by users and $\rho, d$, and $d_{1}$ are same parameters which are designed before. This strategy is a combination of first two strategies. From (38), it can be seen that when the value of control signal $|u(t)|$ is less than $D$, the relative strategy is applied; therefore the system will obtain a more accurate control. While when the value of $|u(t)|$ is high, the control method switches to the fixed threshold strategy so that the error $e(t)$ will ensure the system performance. Apparently, the advantages of the fixed threshold strategy and the relative strategy are brought to this strategy.

In this strategy, it can be obtained that

$$
\bar{e}=\sup |e(t)| \leq \max \left\{\rho D+d_{1}, d\right\} \quad \forall t \in\left[t_{k}, t_{k+1}\right)
$$

Combining the switching threshold strategy above and (39), the following results are stated.
Theorem 4. Consider a certain of nonlinear closed-loop system (1) under Assumption 1 and a switching adaptive controller between the fixed threshold and relative threshold with a userdesigned parameter $D$ with $\bar{d} \geq \bar{e}$. The system is globally stable; that is, all the signals are bounded. And the system can be adjusted by selecting proper parameters. In addition, there must be a time $t^{*}>0$ such that $t^{*}$ is greater than or equal to the execution period $\left\{t_{k+1}-t_{k}\right\}, k \in \mathbb{Z}^{+}$, which means the Zeno behavior is successfully avoided.

Proof. Since the same control law of the first two strategies are adopted by the switching threshold strategy, the system is also globally stable, which means all the signals are bounded. And by letting $|\dot{w}| \leq$, the interexecution $t^{*}$ satisfies $t^{*} \geq$ $\left(\max \left\{d_{1}, d\right\}\right) / \mathbb{k}$. That completes the proof.

\section{Simulation Results}

Example 1. In this section, based on the system given in [49], we combine zero dynamics with it. A stabilization case is illustrated with three proposed event-triggered strategies and time-triggered strategy, and each strategy's performance is compared. Consider the following systems:

$$
\begin{aligned}
\dot{\eta} & =f_{0}(\eta)+g_{0}(\eta) x_{1} \\
\dot{x}_{1} & =x_{2}+\theta x_{1}^{2} \\
\dot{x}_{2} & =u \\
y & =x_{1}
\end{aligned}
$$

where $\theta$ denotes an unknown parameter. Our control goal is now to make the system output $y=x_{1}(t)$ asymptotically back to zero. We use the above four strategies to balance the performance of the system and the times of triggering events. The design constants are chosen as follows: (1) Fixed threshold strategy: $d=3, \bar{d}=4$. (2) Relative threshold strategy: $\rho=0.15, d_{1}=0.3, \bar{d}_{1}=2$. (3) Switching threshold strategy: $D=30$. (4) Time-triggered strategy: interexecution time: $0.05 \mathrm{~s}$. The other parameters are chosen as $x_{1}(0)=$ $0, x_{2}(0)=0, \hat{\theta}(0)=0, \theta=5, c_{1}=7, c_{2}=10, \Gamma=1, \varepsilon=$ 10. And since $f_{0}(\eta)$ and $g_{0}(\eta)$ are known, we define that $f_{0}(\eta)=-\eta, g_{0}(\eta)=1$. The Lyapunov function is chosen as $V_{\eta}=(1 / 2) \eta^{2}$; therefore we get

$$
\dot{V}_{\eta}=\eta \dot{\eta} \leq \eta\left(-\eta+x_{1}\right) \leq \eta\left(-\eta+z_{1}+\alpha_{0}\right)
$$

Let $\alpha_{0}=-c_{0} \eta$. Therefore

$$
\dot{V}_{\eta}=-\left(1+c_{0}\right) \eta^{2}+\eta z_{1}
$$

and $c_{0}$ is chosen as $c_{0}=50$.

Figure 1 shows the stabilization performance of the output signal $y(t)$ in three event-triggered strategies and timetriggered strategy, and the control signals $u(t)$ and $w(t)$ are shown in Figure 2. The times of triggering events is shown in Table 1, while state signal $x_{2}$ and the parameter estimate $\hat{\theta}$ are, respectively, shown in Figures 3 and 4 . It 


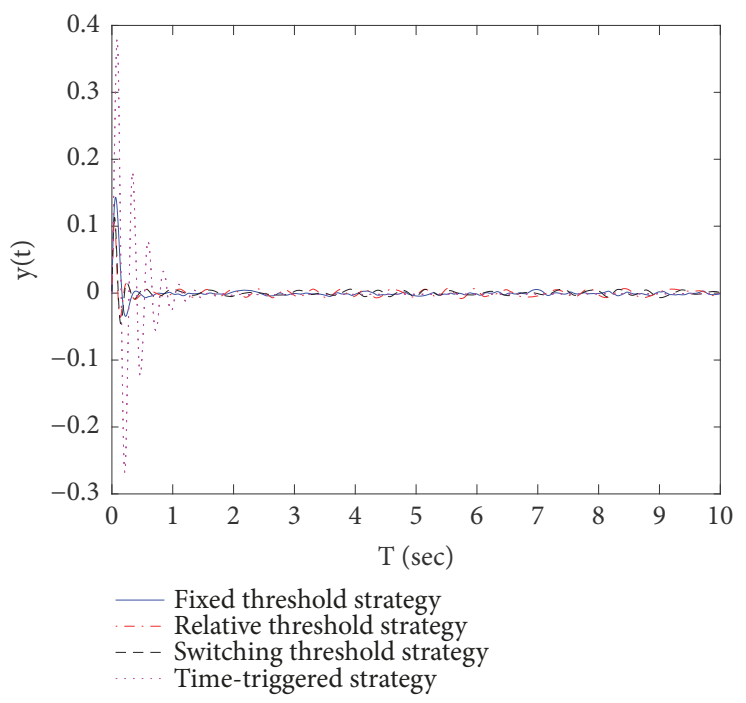

FIgURE 1: Output $y(t)$.

TABLE 1: Times of triggering events.

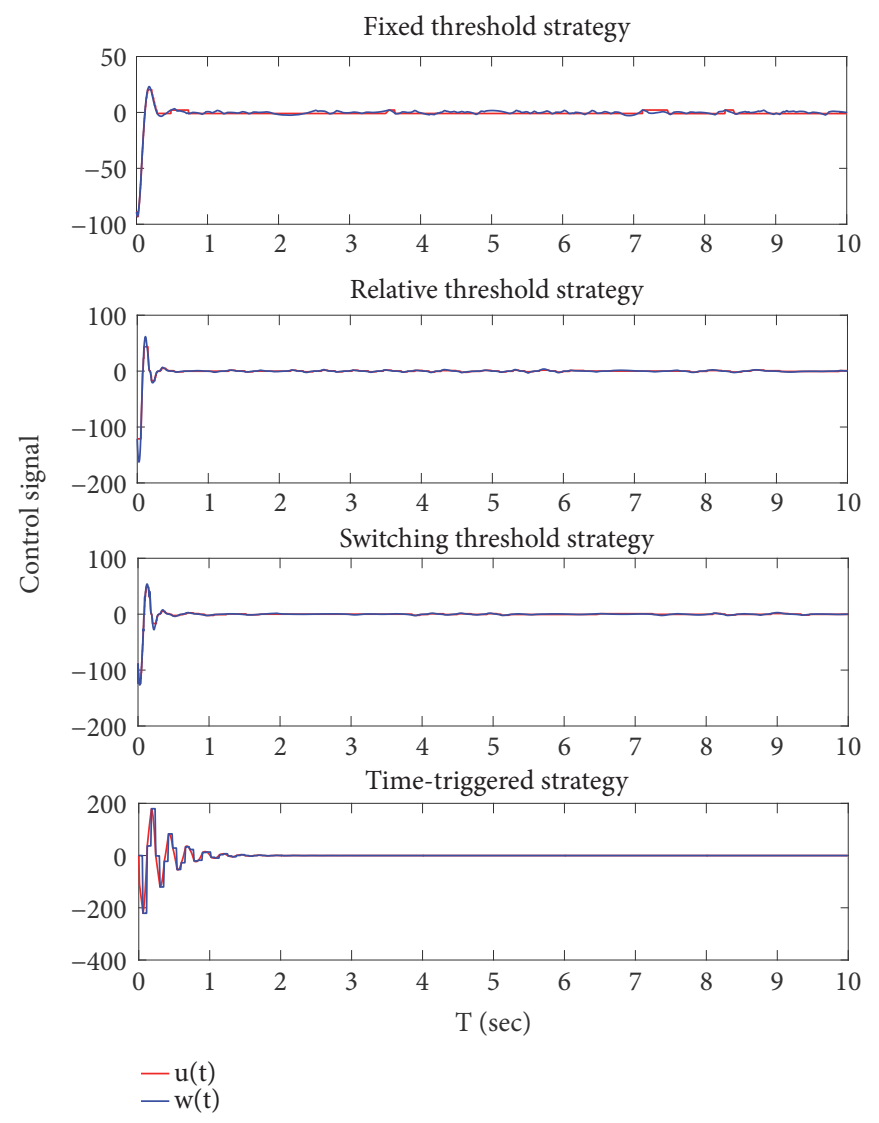

FIGURe 2: Control signal. 


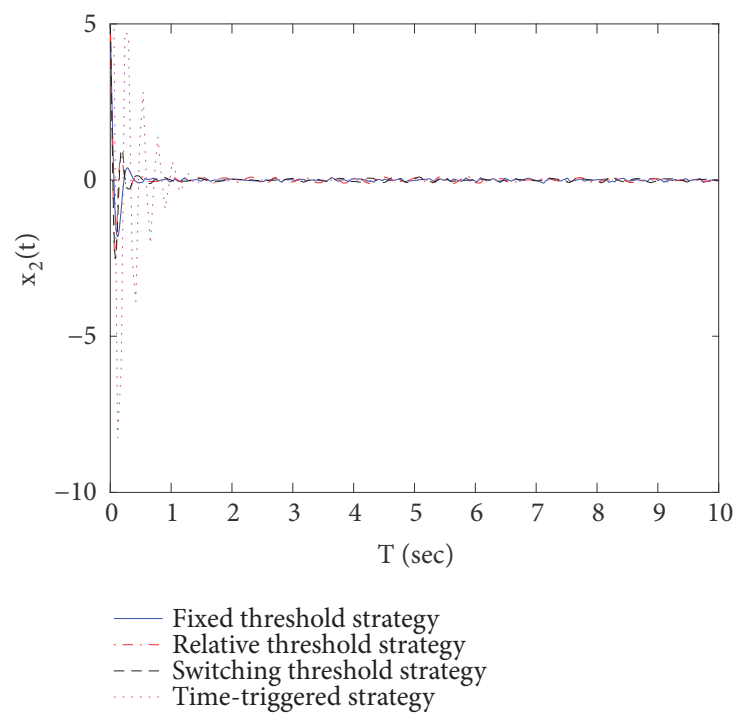

FIgURE 3: State signal $x_{2}(t)$.

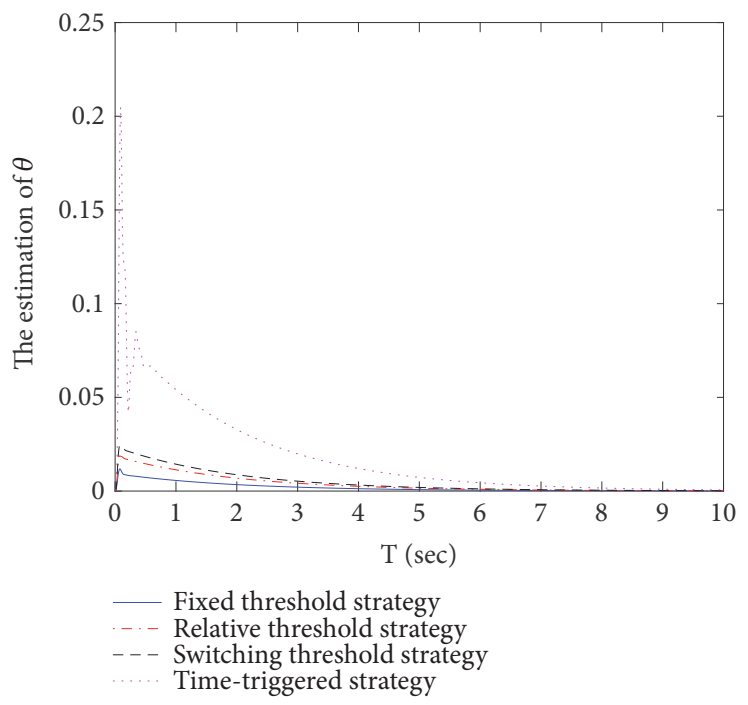

FIgURE 4: Parameter estimate $\widehat{\theta}$.

can be seen that the fixed threshold strategy has the best stabilization performance due to its relatively small threshold, but it has the most triggering events. On the contrary, the relative threshold strategy's performance is not relatively good, while it has the fewest triggering events. And the switching threshold strategy gives a flexibility between the performance of system and times of triggering events. For time-triggered strategy, though it has the best performance at last, it also has the most triggering times, which may result in unnecessary high workloads in practical systems. Besides, the system does not perform well at the beginning.

Example 2. Consider a higher order networked-control system based on [45] with zero dynamics

$$
\dot{\eta}=f_{0}(\eta)+g_{0}(\eta) x_{1}
$$

$$
\begin{aligned}
\dot{x}_{1} & =x_{2}+x_{1}^{2} \\
\dot{x}_{2} & =\theta x_{3} \\
\dot{x}_{3} & =x_{1}+x_{2}+u \\
y & =x_{1}
\end{aligned}
$$

where $\theta$ denotes an unknown parameter. The above three event-triggered strategies are applied to the system. The design constants are chosen as follows: (1) Fixed threshold strategy: $d=10, \bar{d}=11$. (2) Relative threshold strategy: $\rho=$ $0.15, d_{1}=5, \bar{d}_{1}=8$. (3) Switching threshold strategy: $D=$ 60 . The other parameters are chosen as $x_{1}(0)=0, x_{2}(0)=$ $-1, x_{3}(0)=5, \hat{\theta}(0)=0, \theta=1, c_{0}=50, c_{1}=10, c_{2}=$ 


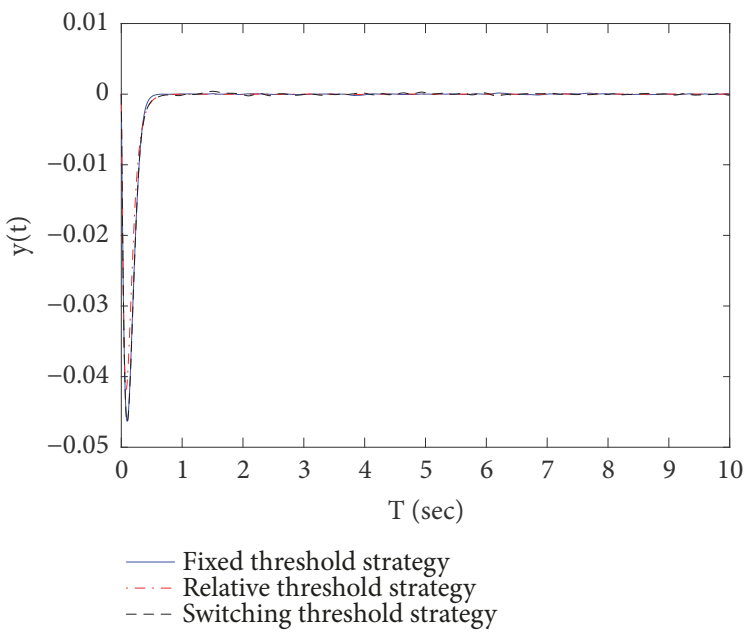

Figure 5: Output $y(t)$.

Fixed threshold strategy
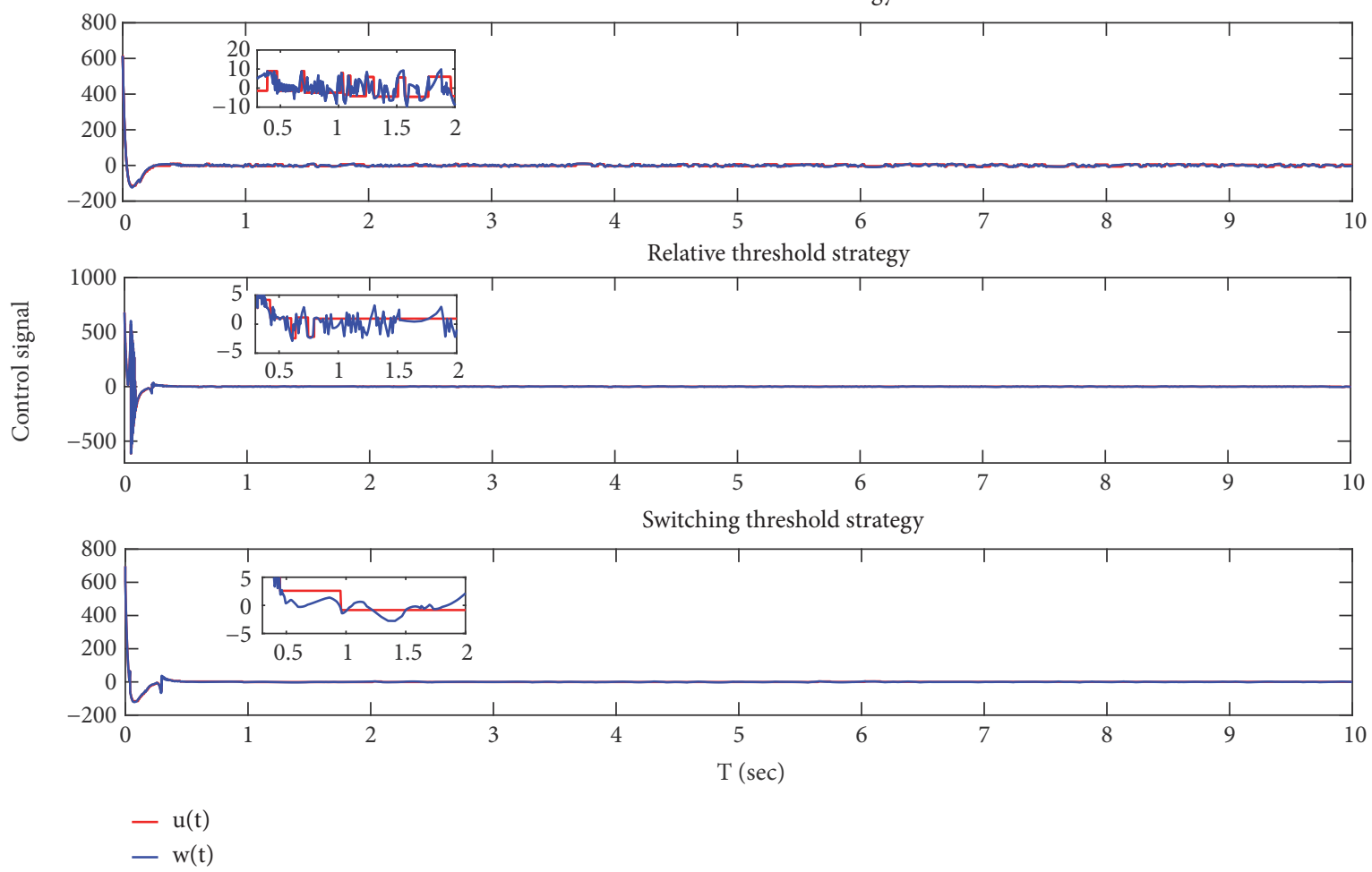

Figure 6: Control signal.

20, $\Gamma=1, \varepsilon=1$. And $f_{0}(\eta)$ and $g_{0}(\eta)$ are defined as $f_{0}(\eta)=$ $\eta^{2}, g_{0}(\eta)=\eta$.

Figure 5 shows the stabilization performance of the output signal $y(t)$ in three event-triggered strategies, and the control signals $u(t)$ and $w(t)$ are shown in Figure 6. The time of triggering events is shown in Table 2, while state signals $x_{2}$ and $x_{3}$ and the parameter estimate $\hat{\theta}$ are, respectively, shown in Figures 7, 8, and 9. In this simulation example, the switching threshold strategy provides the smallest triggering times, but its performance is not better than the other strategies, while the fixed threshold strategy provides the best performance with the most triggering events. And the relative threshold strategy balances the performance and times of triggering events. It can be seen that the parameters can be designed by users to find the most suitable strategy for different practical systems under different situations. 


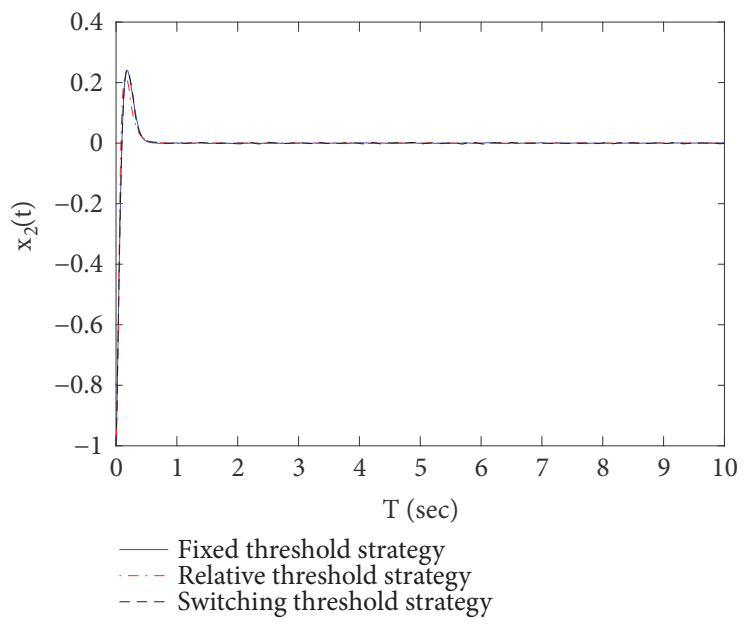

FIgURE 7: State signal $x_{2}(t)$.

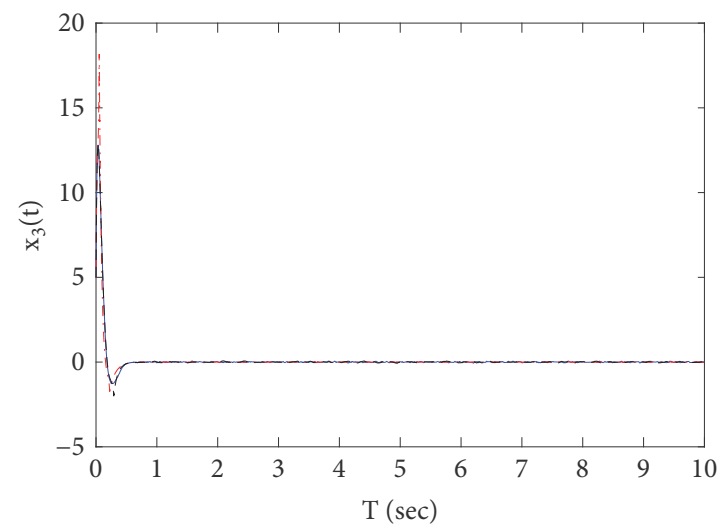

Fixed threshold strategy

Relative threshold strategy

- - Switching threshold strategy

FIgURE 8: State signal $x_{3}(t)$.

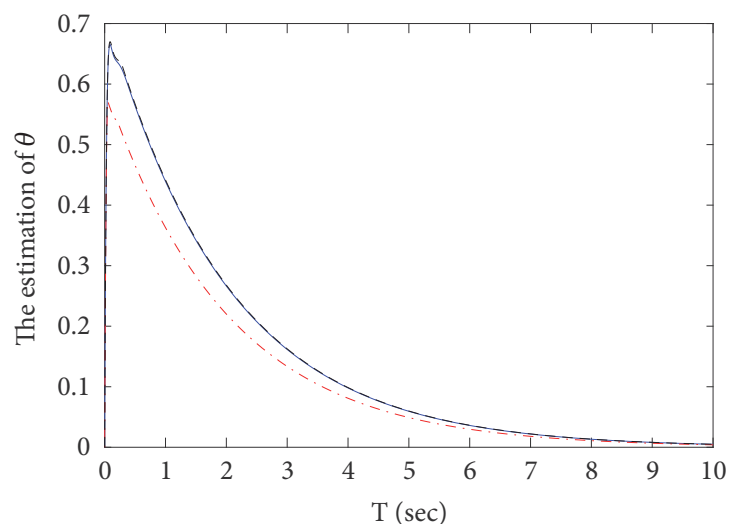

_ Fixed threshold strategy

-..- Relative threshold strategy

- - Switching threshold strategy

Figure 9: Parameter estimate $\widehat{\theta}$. 
TABLE 2: Times of triggering events.

\begin{tabular}{lcc}
\hline Fixed strategy & Relative strategy & Switching strategy \\
\hline 133 & 128 & 82 \\
\hline
\end{tabular}

\section{Conclusion}

This paper has considered an event-triggered adaptive backstepping control for strict-feedback nonlinear systems with zero dynamics. An adaptive control method is utilized to overcome the unknown system parameters, by which all the closed-loop signals are guaranteed to be uniformly bounded. Besides, to overcome the need of ISS assumption, an adaptive controller and event-triggered technique are designed at the same time. And three different event-triggered control strategies are also given. It is shown from the simulation results that all the strategies guarantee that the stability error approaches to a small neighborhood of the origin.

\section{Data Availability}

The data used to support the findings of this study are available from the corresponding author upon request.

\section{Conflicts of Interest}

The authors declare that they have no conflicts of interest.

\section{Acknowledgments}

This work was supported in part by the Taishan Scholar Project of Shandong Province of China under Grant 2015162 and Grant tsqn201812093.

\section{References}

[1] J. Zhou, C. Wen, and W. Wang, "Adaptive control of uncertain nonlinear systems with quantized input signal," Automatica, vol. 95, pp. 152-162, 2018.

[2] Y. Wang, Z. Jia, and Z. Zuo, "Dynamic event-triggered and selftriggered output feedback control of networked switched linear systems," Neurocomputing, vol. 314, pp. 39-47, 2018.

[3] K. P. Tee and S. S. Ge, "Control of nonlinear systems with full state constraint using a barrier lyapunov function," in Proceedings of the 48th IEEE Conference on Decision and Control Held Jointly with 28th Chinese Control Conference (CDC/CCC '09), pp. 8618-8623, Shanghai, China, December 2009.

[4] Y. D. Landau, "Adaptive control: the model reference approach," IEEE Transactions on Systems Man \& Cybernetics, pp. 169-170, 1984.

[5] S. Zheng and W. Li, "Adaptive control for switched nonlinear systems with coupled input nonlinearities and state constraints," Information Sciences, vol. 462, pp. 331-356, 2018.

[6] T. Sun and Y. Pan, "Robust adaptive control for prescribed performance tracking of constrained uncertain nonlinear systems," Journal of The Franklin Institute, vol. 356, no. 1, pp. 18-30, 2019.

[7] X. Xia and T. Zhang, "Adaptive quantized output feedback DSC of uncertain systems with output constraints and unmodeled dynamics based on reduced-order K-filters," Neurocomputing, vol. 310, pp. 236-245, 2018.

[8] C. Yin, Y. Chen, and S. M. Zhong, "Fractional-order sliding mode based extremum seeking control of a class of nonlinear systems," Automatica, vol. 50, no. 12, pp. 3173-3181, 2014.

[9] S. Li, X. Peng, Y. Tang, and Y. Shi, "Finite-time synchronization of time-delayed neural networks with unknown parameters via adaptive control," Neurocomputing, vol. 308, pp. 65-74, 2018.

[10] C. Yin, X. Huang, Y. Chen, S. Dadras, S.-M. Zhong, and Y. Cheng, "Fractional-order exponential switching technique to enhance sliding mode control," Applied Mathematical Modelling: Simulation and Computation for Engineering and Environmental Systems, vol. 44, pp. 705-726, 2017.

[11] A. Ayadi, M. Smaoui, S. Aloui, S. Hajji, and M. Farza, "Adaptive sliding mode control with moving surface: Experimental validation for electropneumatic system," Mechanical Systems and Signal Processing, vol. 109, pp. 27-44, 2018.

[12] C. Chen, Z. Liu, K. Xie, Y. Zhang, and C. . Chen, "Asymptotic adaptive control of nonlinear systems with elimination of overparametrization in a Nussbaum-like design," Automatica, vol. 98, pp. 277-284, 2018.

[13] J. Zhou and W. Wang, "Adaptive Control of Quantized Uncertain Nonlinear Systems," IFAC-PapersOnLine, vol. 50, no. 1, pp. 10425-10430, 2017.

[14] M. Chen and S. Ge, "Adaptive neural output feedback control of uncertain nonlinear systems with unknown hysteresis using disturbance observer," IEEE Transactions on Industrial Electronics, 2015.

[15] C.-Y. Chen, W.-H. Gui, Z.-H. Guan, R.-L. Wang, and S.-W. Zhou, "Adaptive neural control for a class of stochastic nonlinear systems with unknown parameters, unknown nonlinear functions and stochastic disturbances," Neurocomputing, vol. 226, pp. 101-108, 2017.

[16] D. Li and D. Li, "Adaptive neural tracking control for nonlinear time-delay systems with full state constraints," IEEE Transactions on Systems, Man, and Cybernetics: Systems, vol. 47, no. 7, pp. 1590-1601, 2017.

[17] Y. Li, S. Sui, and S. Tong, "Adaptive fuzzy control design for stochastic nonlinear switched systems with arbitrary switchings and unmodeled dynamics," IEEE Transactions on Cybernetics, vol. 47, no. 2, pp. 403-414, 2017.

[18] C. Yin, S. Dadras, X. Huang, J. Mei, H. Malek, and Y. Cheng, "Energy-saving control strategy for lighting system based on multivariate extremum seeking with Newton algorithm," Energy Conversion and Management, vol. 142, pp. 504-522, 2017.

[19] K. Johan Åström and B. Bernhardsson, "Comparison of periodic and event based sampling for first-order stochastic systems," IFAC Proceedings Volumes, vol. 32, no. 2, pp. 5006-5011, 1999.

[20] Y.-X. Li and G.-H. Yang, "Event-triggered adaptive backstepping control for parametric strict-feedback nonlinear systems," International Journal of Robust and Nonlinear Control, vol. 28, no. 3, pp. 976-1000, 2018.

[21] L. Xing, C. Wen, Z. Liu, H. Su, and J. Cai, "Event-triggered adaptive control for a class of uncertain nonlinear systems," Institute of Electrical and Electronics Engineers Transactions on Automatic Control, vol. 62, no. 4, pp. 2071-2076, 2017.

[22] X. Su, F. Xia, J. Liu, and L. Wu, "Event-triggered fuzzy control of nonlinear systems with its application to inverted pendulum systems," Automatica, vol. 94, pp. 236-248, 2018. 
[23] P. Tabuada, "Event-triggered real-time scheduling of stabilizing control tasks," IEEE Transactions on Automatic Control, vol. 52, no. 9, pp. 1680-1685, 2007.

[24] X. Wang and M. D. Lemmon, "Event-triggering in distributed networked control systems," Institute of Electrical and Electronics Engineers Transactions on Automatic Control, vol. 56, no. 3, pp. 586-601, 2011.

[25] Y. Li, H. Li, and W. Sun, "Event-triggered control for robust set stabilization of logical control networks," Automatica, vol. 95, pp. 556-560, 2018.

[26] Z.-G. Wu, Y. Xu, Y.-J. Pan, H. Su, and Y. Tang, "Event-triggered control for consensus problem in multi-agent systems with quantized relative state measurement and external disturbance," IEEE Transactions on Circuits and Systems I: Regular Papers, vol. 65, no. 7, pp. 2232-2242, 2018.

[27] Z.-G. Wu, Y. Xu, Y.-J. Pan, P. Shi, and Q. Wang, "Eventtriggered pinning control for consensus of multiagent systems with quantized information," IEEE Transactions on Systems, Man, and Cybernetics: Systems, vol. 48, no. 11, pp. 1929-1938, 2018.

[28] Z.-G. Wu, Y. Xu, R. Lu, Y. Wu, and T. Huang, "EventTriggered Control for Consensus of Multiagent Systems with Fixed/Switching Topologies," IEEE Transactions on Systems, Man, and Cybernetics: Systems, vol. 48, no. 10, pp. 1736-1746, 2018.

[29] S. Liu and L. Zhou, "Network synchronization and application of chaotic Lur'e systems based on event-triggered mechanism," Nonlinear Dynamics, vol. 83, no. 4, pp. 2497-2507, 2016.

[30] M. Li, J. Zhao, J. Xia, G. Zhuang, and W. Zhang, "Extended dissipative analysis and synthesis for network control systems with an event-triggered scheme," Neurocomputing, vol. 312, pp. 34-40, 2018.

[31] Y. Zhu, X. Guan, X. Luo, and S. Li, "Finite-time consensus of multi-agent system via nonlinear event-triggered control strategy," IET Control Theory \& Applications, vol. 9, no. 17, pp. 2548-2552, 2015.

[32] A. Fu and J. Mazo, "Decentralized periodic event-triggered control with quantization and asynchronous communication," Automatica, vol. 94, pp. 294-299, 2018.

[33] X. Xiao, J. H. Park, and L. Zhou, "Event-triggered control of discrete-time switched linear systems with packet losses," Applied Mathematics and Computation, vol. 333, pp. 344-352, 2018.

[34] Y.-X. Li and G.-H. Yang, "Model-based adaptive event-triggered control of strict-feedback nonlinear systems," IEEE Transactions on Neural Networks and Learning Systems, vol. 29, no. 4, pp. 1033-1045, 2018.

[35] R. Postoyan, P. Tabuada, D. Nesic, and A. Anta, "A framework for the event-triggered stabilization of nonlinear systems," IEEE Transactions on Automatic Control, vol. 60, no. 4, pp. 982-996, 2015.

[36] W. Heemels, K. H. Johansson, and P. Tabuada, "An introduction to event-triggered and self-triggered control," in Proceedings of the 51st IEEE Conference on Decision and Control, 2012.

[37] A. Adaldo, F. Alderisio, D. Liuzza et al., "Event-triggered pinning control of switching networks," IEEE Transactions on Control of Network Systems, vol. 2, no. 2, pp. 204-213, 2015.

[38] A. Isidori, Nonlinear Control Systems, Springer, 1999.

[39] M. Ishitobi, M. Nishi, and S. Kunimatsu, "Stability of zero dynamics of sampled-data nonlinear systems," IFAC Proceedings Volumes, vol. 41, no. 2, pp. 5969-5973, 2008.
[40] D. S. Laila, D. Nesic, and A. Astolfi, "Sampled-data control of nonlinear systems," in Advanced Topics in Control Systems Theory, vol. 328 of Lect. Notes Control Inf. Sci., pp. 91-137, Springer, 2006.

[41] T. Berger and T. Reis, "Zero dynamics and funnel control for linear electrical circuits," Journal of The Franklin Institute, vol. 351, no. 11, pp. 5099-5132, 2014.

[42] D. Hernandez, F. Castanos, and L. Fridman, "Zero-dynamics design and its application to the stabilization of implicit systems," Systems Control Letters, vol. 98, pp. 74-78, 2016.

[43] C. Hua, K. Li, and X. Guan, "Decentralized event-triggered control for interconnected time-delay stochastic nonlinear systems using neural networks," Neurocomputing, vol. 272, pp. 270-278, 2018.

[44] K. J. Åström, P. Hagander, and J. Sternby, “Zeros of sampled systems," Automatica, vol. 20, no. 1, pp. 31-38, 1984.

[45] H. K. Khakil, Nonlinear Systems, Prentice Hall, 2002.

[46] J. Zhou and C. Wen, Adaptive Backstepping Control of Uncertain Systems, vol. 372, Springer, Berlin, Germany, 2008.

[47] P. A. Ioannou, Adaptive Systems with Reduced Models, Lecture Notes in Control and Information Sciences, 1983.

[48] J. P. LaSalle, "Some extensions of Liapunov's second method," IRE Transactions on Circuit Theory, vol. 7, no. 4, pp. 520-527, 1960.

[49] M. Krstic, P. V. Kokotovic, and I. Kanellakopoulos, Nonlinear and Adaptive Control Design, Wiley, 1995. 


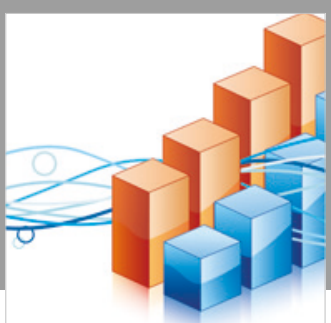

Advances in

Operations Research

\section{-n-m}
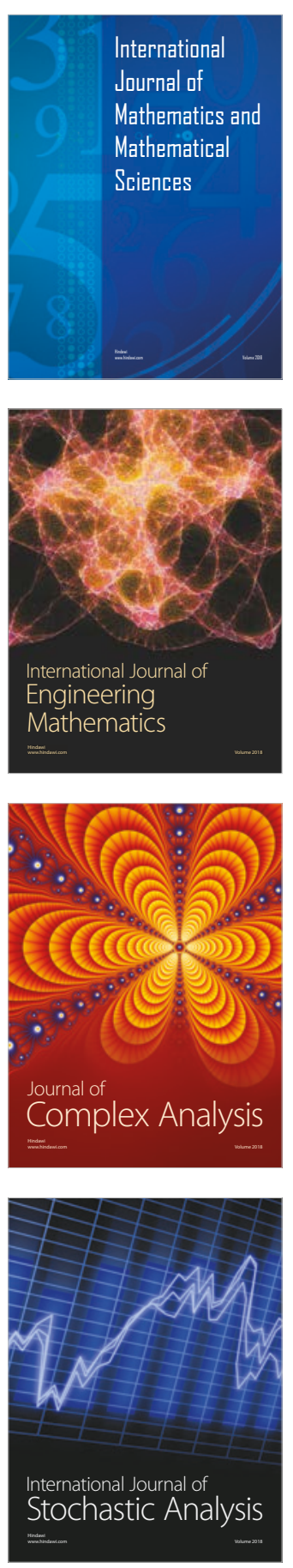
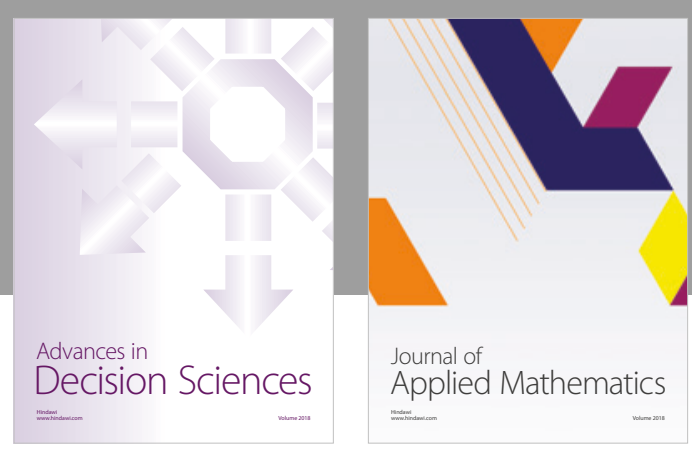

Journal of

Applied Mathematics
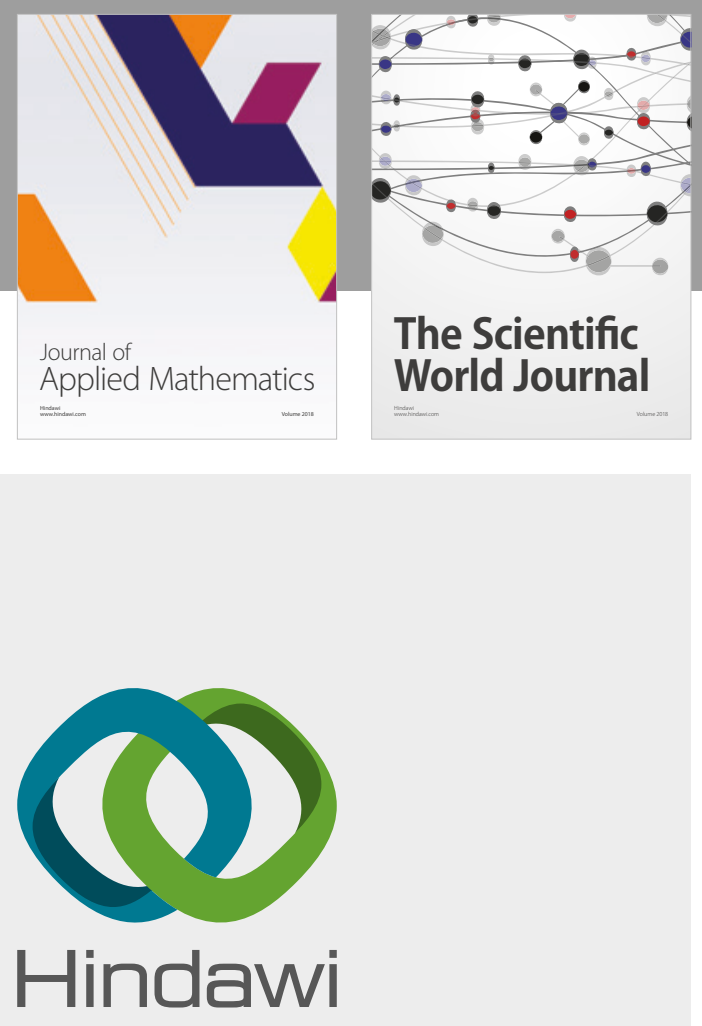

Submit your manuscripts at

www.hindawi.com

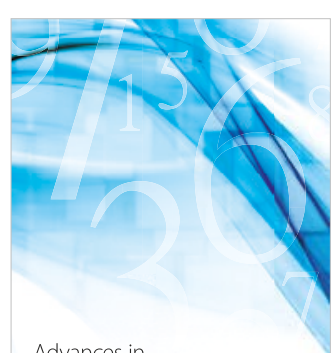

Advances in
Numerical Analysis
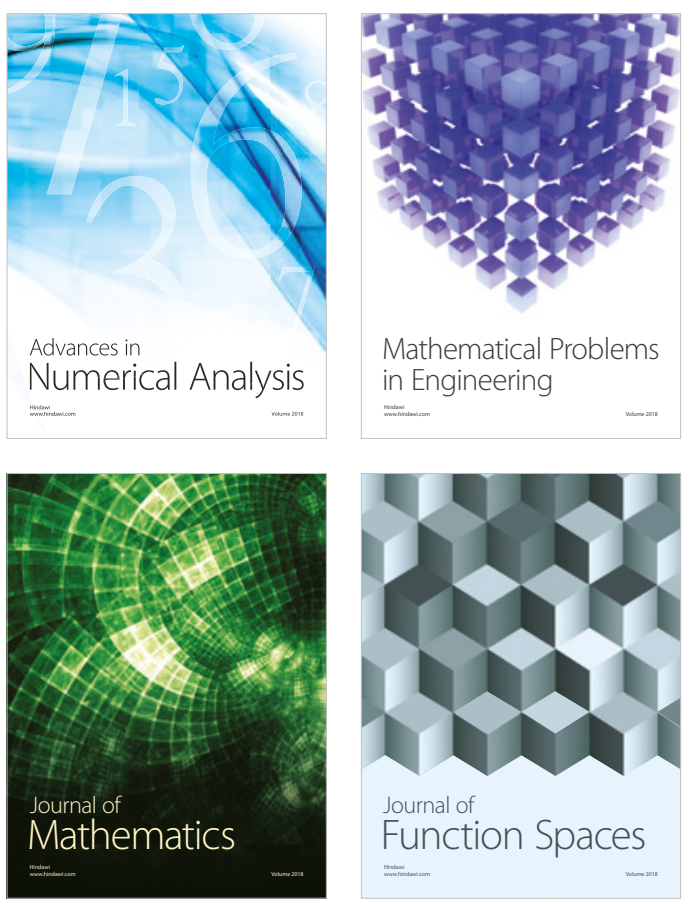

Mathematical Problems in Engineering

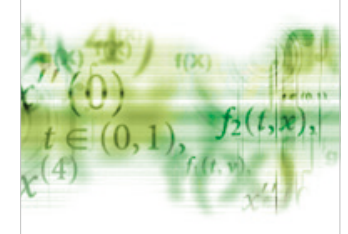

International Journal of

Differential Equations

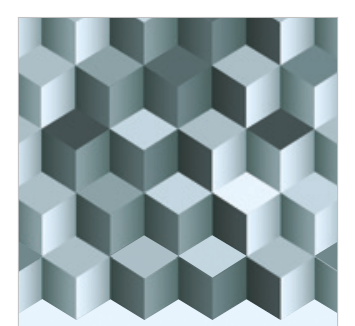

Journal of

Function Spaces
The Scientific

World Journal

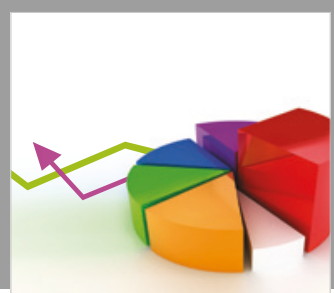

Journal of

Probability and Statistics
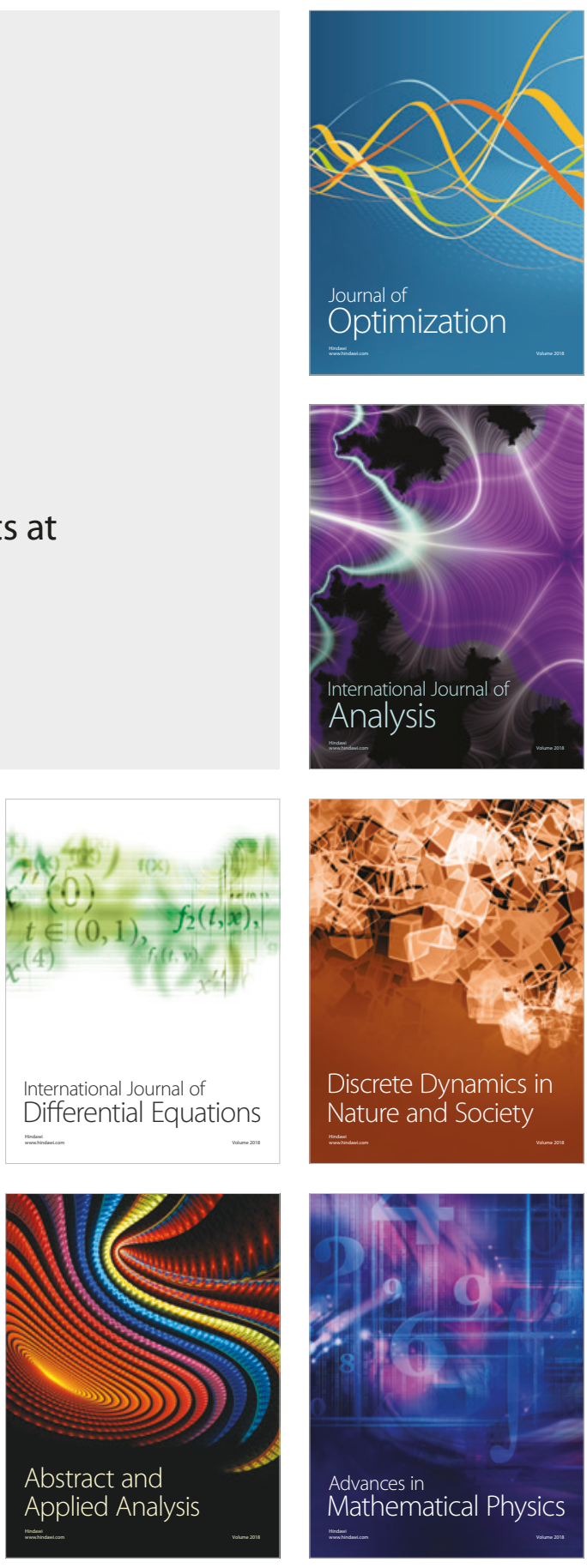